\title{
Note to the Second Edition
}

ThIs revision comes five years and over 500 cases after the first edition. The most gratifying aspect of the first edition was to hear from many of the people who in reading it found comfort, guidance, and knowledge. I hope the second edition will have the same results. 
